\title{
Gain length fitting formula for free-electron lasers with strong space-charge effects
}

\author{
G. Marcus, E. Hemsing, and J. Rosenzweig \\ Particle Beam Physics Laboratory, Department of Physics and Astronomy, University of California Los Angeles, \\ Los Angeles, California 90095, USA
}

(Received 31 May 2011; published 23 August 2011)

\begin{abstract}
We present a power-fit formula, obtained from a variational analysis using three-dimensional freeelectron laser theory, for the gain length of a high-gain free-electron laser's fundamental mode in the presence of diffraction, uncorrelated energy spread, and longitudinal space-charge effects. The approach is inspired by the work of Xie [Nucl. Instrum. Methods Phys. Res., Sect. A 445, 59 (2000)], and provides a useful shortcut for calculating the gain length of the fundamental Gaussian mode of a free-electron laser having strong space-charge effects in the 3D regime. The results derived from analytic theory are in good agreement with detailed numerical particle simulations that also include higher-order space-charge effects, supporting the assumptions made in the theoretical treatment and the variational solutions obtained in the single-mode limit.
\end{abstract}

DOI: 10.1103/PhysRevSTAB.14.080702

PACS numbers: 41.60.Cr, 52.59.Sa

\section{INTRODUCTION}

High-gain free-electron lasers (FELs) operate on the principle that tunable, narrow bandwidth light pulses can be emitted and amplified many orders of magnitude by the strong beam-radiation instability affecting a relativistic electron beam (e-beam) traversing a periodic undulator. The distance along the undulator it takes for the power of the emitted light to increase by a factor of $e$ during the exponential growth regime is known as the power gain length. It is given in the one-dimensional, cold-beam limit as

$$
L_{1 \mathrm{D}}=\frac{1}{2 \sqrt{3} k_{u} \rho},
$$

where $\rho$ is the well-known Pierce parameter [1], given by

$$
\rho=\left[\frac{K[J J]}{4 \sqrt{2}} \frac{\theta_{p} \gamma_{z}}{k_{u} \gamma}\right]^{2 / 3} .
$$

Here, $k_{u}$ is the undulator wave number, $K=e B_{u} / m c k_{u}$ is the dimensionless undulator parameter, with peak magnetic field $B_{u}$. The Lorentz factor $\gamma$ is the electron beam energy in units of the rest energy $m c^{2}$, while the Lorentz factor relating the average longitudinal beam motion to the laboratory frame is $\gamma_{z}=\gamma / \sqrt{1+\frac{K^{2}}{2}}$. The factor accounting for the coupling of the electron motion to radiation emission in the case of a planar undulator is $[J J]=$ $J_{0}\left[K^{2} /\left(4+2 K^{2}\right)\right]-J_{1}\left[K^{2} /\left(4+2 K^{2}\right)\right]$, where $J_{0}$ and $J_{1}$ are Bessel functions of the first kind. The relativistic plasma wave number is

Published by the American Physical Society under the terms of the Creative Commons Attribution 3.0 License. Further distribution of this work must maintain attribution to the author(s) and the published article's title, journal citation, and DOI.

$$
\theta_{p}=\sqrt{\frac{2 I_{e}}{I_{A} \gamma \gamma_{z}^{2} \sigma_{x}^{2}}},
$$

where $\sigma_{x}$ is the rms electron beam size, $I_{e}$ is the electron beam peak current, and $I_{A} \simeq 17 \mathrm{kA}$ is the Alfvén current.

The power gain length is one of the most important parameters in the design of a high-gain FEL, as it determines the overall size (and thus cost) of the undulator system needed for the FEL to reach saturation. Onedimensional theory, however, does not entirely capture the complexity of high-gain FELs operating in the infrared or visible wavelength region where various strong threedimensional effects can be detrimental to performance and ultimately degrade the gain. For these 3D FELs the gain length $L_{g}$ exhibits a sensitive and complicated dependence on diffraction (the tendency of the light to spread while propagating), the detuning from resonance, and the uncorrelated energy spread and longitudinal space charge in the e-beam. Given the large number of physical parameters that influence the dynamics, it is useful to describe these effects using scaled parameters that individually represent the essential features of the FEL system [2]. Here we focus on the three of highest relevance to optical regime FELs: the diffraction parameter, $\eta_{d}=L_{1 \mathrm{D}} / 2 k \sigma_{x}^{2}$, which quantifies the extent to which transverse effects contribute to the gain for a FEL with wavelength $\lambda=2 \pi / k$; the scaled energy spread parameter $\eta_{\gamma}=2 k_{u} \sigma_{\gamma} L_{1 \mathrm{D}}$, which captures the contribution of the rms uncorrelated e-beam energy spread $\sigma_{\gamma}$; and $\bar{\theta}_{p}=2 \theta_{p} L_{1 \mathrm{D}}$, the space-charge parameter, which is scaled to be twice the plasma phase advance over a one-dimensional gain length. Even in terms of this reduced set of parameters, determination of $L_{g}$ for design and optimization of the FEL system requires either numeric solutions to the governing equations or fully 3D numerical particle simulations, which are often time consuming. Therefore, it would be useful to quickly evaluate 
the performance of the FEL system without having to resort to these more time intensive and computationally costly methods.

For short-wavelength FELs in which emittance effects can play an important role and where space charge can be neglected $\left(\bar{\theta}_{p} \rightarrow 0\right)$, Xie [2] provided a power-fit formula that has proven to be extremely useful for quickly predicting the growth rate of the dominant Gaussian optical mode. The condition of neglecting space charge is usually easily satisfied for these high energy $\left(\gamma \sim 10^{4}\right)$, short-wavelength FELs such as the Linac Coherent Light Source (LCLS) [3] where $\rho \sim 10^{-4}$ and $\bar{\theta}_{p} L_{1 \mathrm{D}} \ll 1$. However, many FELs of current interest do not operate in this regime. The longitudinal space-charge field begins to counteract the microbunching process as $\bar{\theta}_{p}$ nears unity $\left(\theta_{p}\right.$ approaches $\left.L_{1 \mathrm{D}}^{-1}\right)$, which occurs as $\rho \rightarrow \theta_{p} \lambda_{u}$ as evidenced in Eq. (2). Spacecharge effects are of interest for low energy Raman FELs where the beam charge density is sufficiently high [4-6]. The performance of the FEL in this limit is fundamentally changed by collective space-charge effects. Of relevance recently are IR-optical self-amplified spontaneous emission (SASE) high-gain FELs that are based on very high-brightness electron beams at relatively low energy $\left(\gamma \sim 10^{2}\right)$, and thus potentially are susceptible to both space-charge and diffraction effects. For instance, the $\sim 800 \mathrm{~nm}$ VISA FEL [7] was characterized by $\rho \sim 5 \times 10^{-3}$, giving $L_{1 \mathrm{D}} \simeq 10 \mathrm{~cm}$, but with diffraction and space-charge effects the actual measured gain length was $18 \mathrm{~cm}$. While diffraction is always a notable degrading effect to the gain for IR-optical FELs, space charge may often play a larger role than the electron beam energy spread or emittance.

FELs also have long been considered candidates as high average power light sources [8] since the e-beam that acts as the gain medium does not suffer from thermal loading and excitation bandwidth constraints that limit conventional sources. FEL oscillators first demonstrated the progress made in realizing this application [9]. More recently, there has been increased interest in obtaining high average power using high-gain amplifiers operating at $\sim 100 \mathrm{MeV}$ e-beam energies and $I_{e} \sim 1 \mathrm{kA}$ currents where longitudinal space charge will strongly affect the FEL performance $[10,11]$. We note in this regard that the e-beam used in such FELs will operate in a space-charge dominated, rather than emittance dominated, mode, and thus the dominance of space charge over emittance effects in the longitudinal FEL dynamics should not be surprising. Similarly, the investigation of longitudinal space-charge waves in high-brightness beams is still currently of great theoretical interest $[12,13]$, following past emphases on understanding transverse beam-plasma oscillations [14]. With all this in mind, we note that there is currently no handy formulation for quickly predicting important FEL characteristics when space charge has significant influence, despite the fact that there has been strong historical interest and recent experimental investigations into FELs that operate under these conditions. While some progress has been made to this end in [15], the numerical algorithm produced therein suffers the same time-consuming constraints as mentioned previously.

For the above reasons, here we revisit the power-fit approach of Xie, including in the fit analysis space charge as a relevant parameter while ignoring the negligible effect of e-beam emittance, $\epsilon_{x}$, in the limit that $\eta_{\epsilon}=$ $2 k L_{1 \mathrm{D}} \epsilon_{x}^{2} / \sigma_{x}^{2} \rightarrow 0$, or $\eta_{\epsilon} \ll 2 \eta_{\gamma}$ in the presence of energy spread, and $\sqrt{\eta_{\epsilon} \eta_{d}} \ll 1$. We follow the analysis of $[16,17]$ and solve the three-dimensional integro-differential FEL equations for the FEL field amplitudes in the limit where the FEL signal field is dominated by the fundamental Gaussian mode. We express, similar to Xie, the gain length of the three-dimensional mode $L_{g}$ as

$$
\overline{\delta k_{i}}=\frac{L_{1 \mathrm{D}}}{L_{g}}=\frac{1}{1+\Lambda_{0,0}},
$$

where $\overline{\delta k_{i}}$ is maximized at the optimal detuning to yield the shortest possible gain length and $\Lambda_{0,0}$ is expressed as a power fitting formula that is a function of $\eta_{d}, \eta_{\gamma}$, and $\bar{\theta}_{p}$. The fit is obtained from numerical solutions to the analytic theory presented in the next section, and shows excellent overall agreement with results from numerical particle simulations performed with GENESIS 1.3 [18] that also include higher-order space-charge distributions. We emphasize that the results obtained in the following sections are in general limited to FELs that operate from the optical to the far IR, or otherwise satisfy the stated parametric constraints.

\section{ANALYTIC MODEL}

The three-dimensional high-gain FEL equations in the presence of uncorrelated energy spread and non-negligible space-charge effects have been explored in previous work [16]. Under a slowly varying transverse field approximation, $\quad \mathbf{E}_{\perp}(\mathbf{x}, t)=\operatorname{Re}\left[\tilde{\mathbf{E}}_{s}\left(\mathbf{x}_{\perp}, z\right) e^{i k(z-c t)}\right], \quad$ the integrodifferential equation that describes the FEL field amplitude evolution along the undulator is given as

$$
\begin{aligned}
D(z) \tilde{E}_{s}= & i \frac{k \mu_{0} e c^{2}}{\gamma m v_{0}^{2}} \int_{0}^{z} d z^{\prime}\left[\frac{[J J] e K^{2}}{4 \gamma^{2}} \tilde{E}_{s}+\frac{e}{k^{2}} D\left(z^{\prime}\right) \tilde{E}_{s}\right] \\
& \times \int_{-\infty}^{\infty} d \eta \frac{\partial F_{0}}{\partial \eta} e^{i\left[\left(k / \gamma_{z}^{2}\right) \eta-\theta_{0}\right]\left(z^{\prime}-z\right)},
\end{aligned}
$$

where $D(z)=\nabla_{\perp}^{2}+2 i k \frac{\partial}{\partial z}$ is the paraxial wave operator, $\eta=\left(\gamma_{1}-\gamma\right) / \gamma$ is the relative energy deviation of an electron with energy $\gamma_{1}$ from the nominal beam energy $\gamma, F_{0}=F_{0}\left(\mathbf{x}_{\perp}, \eta\right)$ is the unmodulated e-beam distribution which stays fixed during transport, $\theta_{0}=k / \gamma_{z}^{2}-k_{u}$ is the detuning, and $\gamma_{z}^{2}=1 /\left(1-v_{0}^{2} / c^{2}\right)$ is the relativistic longitudinal energy factor. Zero initial modulation in the beam has been assumed, as has the condition $k \sigma_{x} / \gamma_{z} \gg 1$, 
which stipulates that the e-beam profile is large compared to the microbunching wavelength in the moving frame. Under this latter constraint the space-charge fields are assumed to be predominantly longitudinal. Further, to make the analysis more tractable, the transverse variation of the charge density distribution is also neglected.

In the single-mode limit, the FEL is assumed to be dominated by a fixed-profile transverse mode that grows exponentially in amplitude along $z$. The field can then be written simply as

$$
\tilde{E}_{s}=E_{p, l} u_{p, l}\left(\mathbf{x}_{\perp}\right) \exp (i \delta k z),
$$

where $E_{p, l}$ is the mode amplitude and $\delta k=\delta k_{r}-i \delta k_{i}$ is the complex wave number associated with the FEL process. By inserting this into (5), one can calculate the gain length $L_{g}=1 / 2 \delta k_{i}$ of the $(p, l)$ mode in the linear regime, where the dominant, exponentially growing solutions are characterized by $\delta k_{i}>0$. The modal profile distribution $u_{p, l}\left(\mathbf{x}_{\perp}\right)$ is any suitable function with indices $p$ and $l$ to describe the mode of interest.

Following [17], a Laguerre-Gaussian basis provides a convenient description for a FEL with cylindrical symmetry:

$$
u_{p, l}\left(\mathbf{x}_{\perp}\right)=\exp \left[i l \phi-\frac{r^{2}}{w^{2}}\right]\left(\frac{r \sqrt{2}}{w}\right)^{|l|} L_{p}^{|l|}\left(\frac{2 r^{2}}{w^{2}}\right),
$$

where $L_{p}^{|l|}$ is a Laguerre polynomial. With an uncorrelated Gaussian e-beam distribution, $F_{0}=n_{0}\left(\sqrt{2 \pi \sigma_{\gamma}^{2}}\right)^{-1} \times$ $\exp \left[-r^{2} / 2 \sigma_{x}^{2}-\eta^{2} / 2 \sigma_{\gamma}^{2}\right]$, Eq. (5) reduces in the singlemode limit to an expression that yields the complex wave number $\delta k$ for the $(p, l)$ mode,

$\left[\overline{S_{1}}-{\overline{\theta_{p}}}^{2} \mathbb{F}_{p, l}\right]\left[\overline{\delta k}+\frac{\eta_{d}}{\alpha}(2 p+|l|+1)\right]=-\left(\frac{2}{\sqrt{3}}\right)^{3} \mathbb{F}_{p, l}$.

Scaled variables have been introduced: $\overline{\delta k}=2 L_{1 \mathrm{D}} \delta k$ is the scaled complex wave number and $\alpha^{2}=w^{2} / 4 \sigma_{x}^{2}$ is the complex spot size parameter. The energy spread contribution to the gain is given by the term

$$
\overline{S_{1}}=-2 \sqrt{2 \pi} \eta_{\gamma}^{3}\left[\int d \bar{\eta} \frac{\bar{\eta} \exp \left(-\bar{\eta}^{2} / 2 \eta_{\gamma}^{2}\right)}{\overline{\delta k}-\bar{\theta}+2 \bar{\eta}}\right]^{-1},
$$

where $\bar{\theta}=2 L_{1 \mathrm{D}} \theta_{0}$. The coupling between the optical modes and the transversely Gaussian e-beam profile is given by

$$
\begin{aligned}
\mathbb{F}_{p, l}= & \frac{(2 p+|l|) !}{p !(p+|l|) !} \frac{\alpha^{2 p}}{(\alpha+1)^{2 p+|l|+1}} \\
& \times{ }_{2} F_{1}\left(-p ;-p ;-2 p-|l| ; 1-\frac{1}{\alpha^{2}}\right),
\end{aligned}
$$

where ${ }_{2} F_{1}(a ; b ; c ; x)$ is the hypergeometric function.

By virtue of the $\bar{\theta}_{p}^{2} \mathbb{F}_{p, l}$ term in (8), the effect of longitudinal plasma oscillations for higher-order FEL modes are included. This is a quasi-three-dimensional extension of a purely one-dimensional model that takes into account, as a first-order approximation, the modification to the spacecharge field profile due to the structure of the optical modes that map to the transverse microbunching profile. Averaged over the beam, this modifies the effective plasma wave number by the coupling factor $\mathbb{F}_{p, l}$.

In general, solutions to (8) can be found for a mode $(p, l)$ by application of the variational condition $\delta(\overline{\delta k}) / \delta \alpha=0$ as in $[2,19]$. The scaled complex wave number $\overline{\delta k}$ and spot size parameter $\alpha$ can then be determined by solving the two resulting equations.

\section{A. One-dimensional limit}

In the 1D limit, the transverse variation of the e-beam and the fields is neglected. The diffraction parameter becomes vanishingly small $\eta_{d} \rightarrow 0$, and the degeneracy of the modes sends the coupling factor to unity $\mathbb{F}_{p, l} \rightarrow 1$. Equation (8) is then given simply as

$$
\left[\overline{S_{1}}-{\overline{\theta_{p}}}^{2}\right] \overline{\delta k}=-\left(\frac{2}{\sqrt{3}}\right)^{3} \text {. }
$$

In the additional limit of vanishing energy spread for a cold beam, $\eta_{\gamma} \rightarrow 0$, Eq. (9) reduces to $\overline{S_{1}}=(\overline{\delta k}-\bar{\theta})^{2}$ and we obtain the familiar cubic equation for $\overline{\delta k}$ of the 1D highgain FEL,

$$
\left[(\overline{\delta k}-\bar{\theta})^{2}-\bar{\theta}_{p}^{2}\right] \overline{\delta k}=-\left(\frac{2}{\sqrt{3}}\right)^{3} .
$$

Figure 1 depicts how the scaled gain of the cold-beam 1D system varies with the detuning for several values of the space-charge parameter [20]. At resonance $(\bar{\theta}=0)$ and in the absence of space-charge effects $\left(\bar{\theta}_{p}=0\right)$ dominant solutions to (12) are simply $\overline{\delta k}=2 L_{1 \mathrm{D}} \delta k=1 / \sqrt{3}-i$, and the $1 \mathrm{D}$ gain length is retrieved from $L_{1 \mathrm{D}}=1 / 2 \delta k_{i}$. In the collective regime where space-charge effects are significant, it is straightforward to show that the shortest gain length (the maximum of $\overline{\delta k}_{i}$ ) is obtained at a detuning

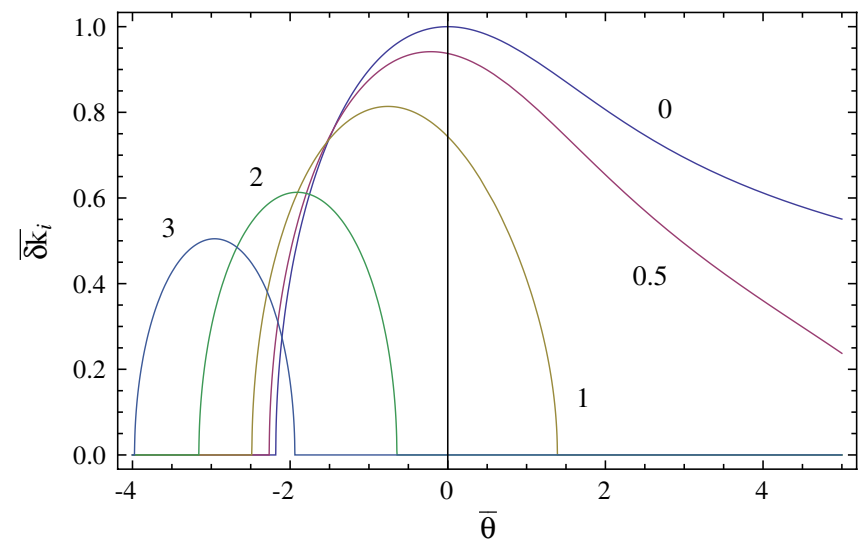

FIG. 1. Detuning gain curves in the 1D limit for $\bar{\theta}_{p}=0,0.5,1$, 2 , and 3 . 


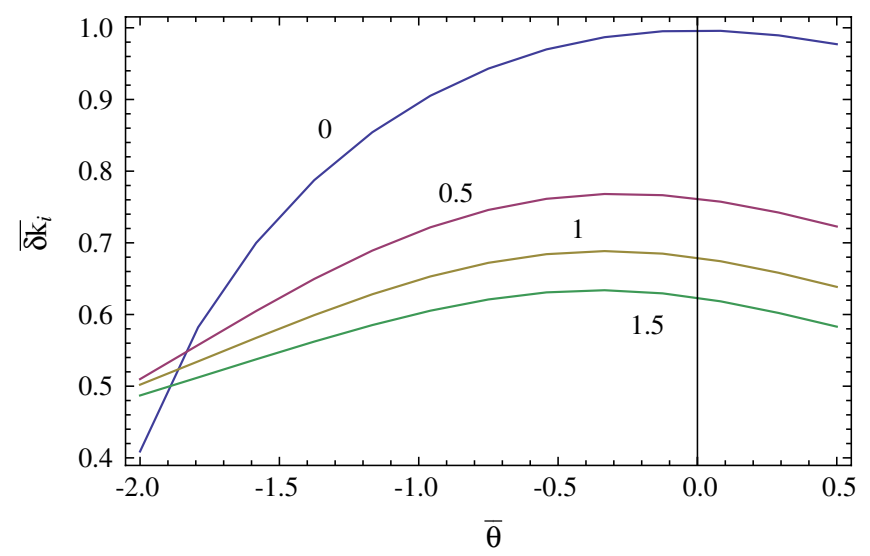

FIG. 2. Detuning curves of the fundamental $(0,0)$ mode for $\eta_{d}=0,0.5,1$, and 1.5 with $\bar{\theta}_{p}, \eta_{\gamma}=0$.

of $\bar{\theta}=-\bar{\theta}_{p}$, in the limit $\overline{\delta k}_{i} \ll \bar{\theta}_{p}$ [21]. The enhanced oscillatory effects of space charge on the FEL-induced charge wave serve to move the frequency into optimized resonance at this detuning.

\section{B. Fundamental mode}

For the fundamental Gaussian mode $(p, l)=(0,0)$, the variational constraint $\delta(\overline{\delta k}) / \delta \alpha=0$ applied to the 3D single-mode expression in (8) yields the equations

$$
\begin{gathered}
\left(\overline{S_{1}}-\frac{{\overline{\theta_{p}}}^{2}}{1+\alpha}\right)\left(\overline{\delta k}+\frac{\eta_{d}}{\alpha}\right)+\left(\frac{2}{\sqrt{3}}\right)^{3} \frac{1}{1+\alpha}=0, \\
-\left(\overline{S_{1}}-\frac{{\overline{\theta_{p}}}^{2}}{1+\alpha}\right) \frac{\eta_{d}\left(1+\alpha^{2}\right)}{\alpha^{2}}+{\overline{\theta_{p}}}^{2}\left(\overline{\delta k}+\frac{\eta_{d}}{\alpha}\right)=\left(\frac{2}{\sqrt{3}}\right)^{3} .
\end{gathered}
$$

Figures 2-4 illustrate how $\eta_{d}, \bar{\theta}_{p}$, and $\eta_{\gamma}$ affect the gain curves of the fundamental mode according to (13). Each contribution pushes the peak of the gain curve into the detuning region $\bar{\theta}<0$ where the e-beam energy is above the resonant energy. The maximum in the detuning curve varies in a complicated way with the parameters that

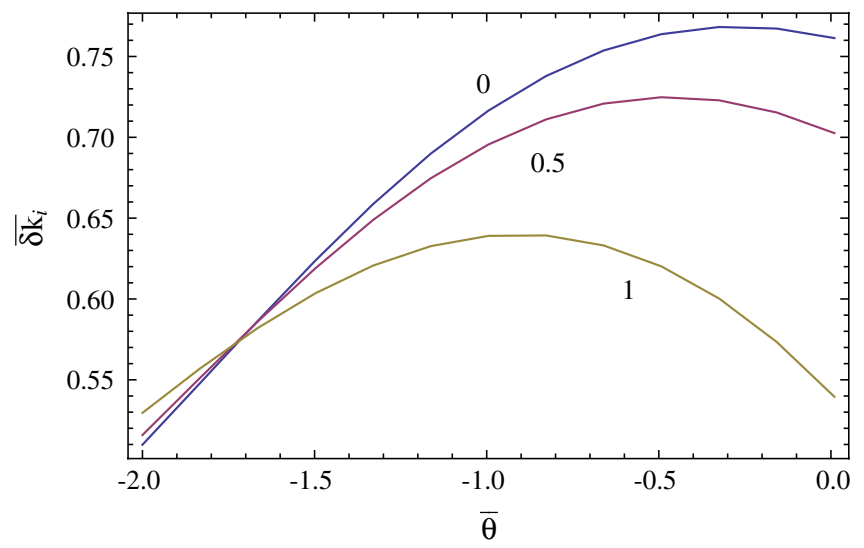

FIG. 3. Contribution of space charge on 3D system: $\bar{\theta}_{p}=0$, 0.5 , and 1 with $\eta_{\gamma}=0, \eta_{d}=0.5$.

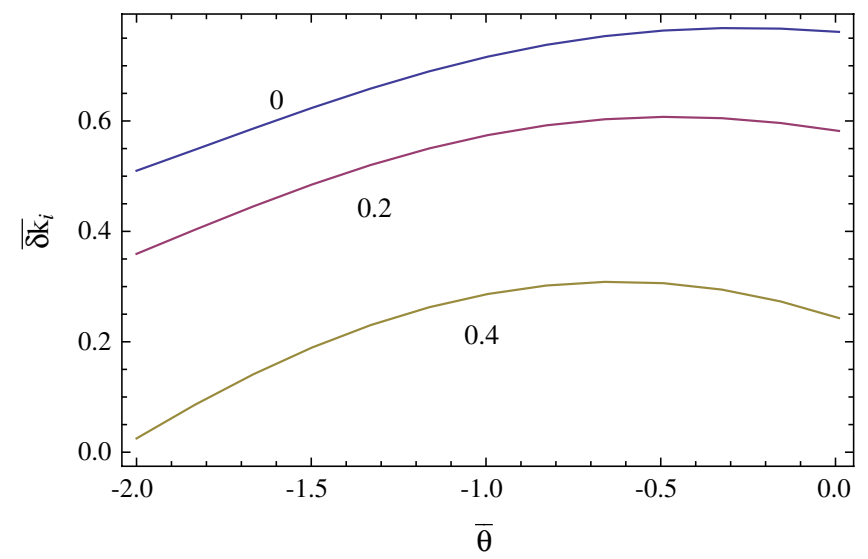

FIG. 4. Contribution of energy spread on 3D system: $\eta_{\gamma}=0$, 0.2 , and 0.4 with $\bar{\theta}_{p}=0, \eta_{d}=0.5$.

specify the FEL. While these effects have been studied previously [20,22], they serve to demonstrate that the desired power-fit formula, calculated over a wide range of accessible parameters that still yield high-gain solutions, would be a useful tool over numerical solutions of the full equations for quick determination of the peak gain.

\section{POWER-FIT FORMULA}

Numerical solutions to the full variational equations taken from Eq. (8) are fit to a power formula over the constituent parameter space of $\eta_{d}, \eta_{\gamma}, \bar{\theta}_{p}$, and $\bar{\theta}$. At the detuning value that minimizes the gain length for given values of $\left(\eta_{d}, \eta_{\gamma}, \bar{\theta}_{p}\right)$, the fit is found to be

$$
\begin{aligned}
\Lambda_{0,0}= & 0.450 \eta_{d}^{0.570}+3.00 \eta_{\gamma}^{2}+0.196 \bar{\theta}_{p}^{1.91} \\
& +51.0 \eta_{d}^{0.950} \eta_{\gamma}^{3}+0.0988 \eta_{d}^{0.230} \bar{\theta}_{p}^{1.21} \\
& +0.0375 \eta_{\gamma}^{0.875} \bar{\theta}_{p}^{12.7}+2.35 \eta_{d}^{11.9} \eta_{\gamma}^{14.9} \bar{\theta}_{p}^{11.4} .
\end{aligned}
$$

We note the precise agreement with Xie's fitting formula for the parameters $\eta_{d}$ and $\eta_{\gamma}$ when $\bar{\theta}_{p}=0$. To illustrate the utility of this formula and in the spirit of Xie we use the representative parameters for the MW class FEL amplifier from [10] where $E=65 \mathrm{MeV}, K=1.26, \lambda_{u}=1.8 \mathrm{~cm}$, $\epsilon_{n}=2.0 \mathrm{~mm} \mathrm{mrad}, \quad I=600 \mathrm{~A}, \quad \sigma_{\gamma}=5 \times 10^{-4}$, and $\sigma_{x}=100 \mu \mathrm{m}$. We find $\eta_{d}=0.641, \eta_{\gamma}=0.028$, and $\bar{\theta}_{p}=0.400$. The effects of diffraction, energy spread, and longitudinal space charge conspire to extend the gain length from $L_{1 \mathrm{D}}=0.081 \mathrm{~m} \rightarrow L_{g}=0.114 \mathrm{~m}$.

\section{SIMULATION AND RESULTS}

The FEL simulation code GENESIS 1.3 [18] was used to evaluate the accuracy of the fitting formula in (14). Nearly 14000 time-independent simulations were performed using a large range of rms transverse electron beam sizes $\left(\sigma_{x}=100-600 \mu \mathrm{m}\right)$, relative rms energy spreads $\left(\sigma_{\gamma}=\right.$ $\left.0-1 \times 10^{-3}\right)$, and beam currents $(I=200-2000 \mathrm{~A})$ in 
TABLE I. Scan parameters. Simulations (theory).

\begin{tabular}{lcc}
\hline \hline Parameter & \multicolumn{1}{c}{ Symbol } & Values \\
\hline Diffraction & $\eta_{d}=L_{1 \mathrm{D}} / 2 k \sigma_{x}^{2}$ & $0.04\left(10^{-4}\right)-0.98(1)$ \\
Energy spread & $\eta_{\gamma}=2 k_{u} \sigma_{\gamma} L_{1 \mathrm{D}}$ & $0\left(10^{-4}\right)-0.34(0.4)$ \\
Space charge & $\bar{\theta}_{p}=2 \theta_{p} L_{1 \mathrm{D}}$ & $0.16\left(10^{-4}\right)-0.42(1)$ \\
\hline \hline
\end{tabular}

order to sample a significant portion of the scaled parameter space where the analytical model is applicable (see Table I). As with the analytic model, the detuning was adjusted to obtain the shortest gain length for each set of parameters. At each optimized detuning value we obtain a scaled value of the gain, denoted by ${\overline{\delta k_{i}}}^{G}$.

To quantify the agreement between the analytic model fit in (14) with results from GENESIS simulations, we define a variance of the form $\sigma^{2}=\frac{1}{N} \sum_{j}^{N}\left(\overline{\delta k}_{i}(j)-\overline{\delta k}_{i, j}^{G}\right)^{2}$, where $\overline{\delta k_{i}}(j)$ is the value of the scaled gain from the analytic fit at the point $\left(\eta_{d, j}, \eta_{\gamma, j}, \bar{\theta}_{p, j}\right)$, and $\overline{\delta k_{i, j}^{G}}$ is the numerical value from GENESIS at the same point. Good agreement is found, with a variance of $\sigma^{2}=5.4 \times 10^{-4}$ over the entire parameter space. For comparison, an independent power fit was performed according to the data points from GENESIS. We denote this fit by the scaled gain function $\overline{\delta k}_{i}^{F}=$ $\overline{\delta k}_{i}^{F}\left(\eta_{d}, \eta_{\gamma}, \bar{\theta}_{p}\right)$. Using the fit function $\overline{\delta k}_{i}^{F}$ in place of the analytic fit function $\overline{\delta k_{i}}$, the variance was nearly the same value, $\sigma^{2}=4 \times 10^{-4}$, demonstrating that the model in (14) provides a reliable measure of the scaled gain length. Figure 5 shows the qualitative consistency between the analytic contour from (14) and data points obtained through GENESIS simulations for the specific case of $\eta_{\gamma}=0$.

Figure 6 shows the relative difference between the analytic and simulated fits, given by $\sigma^{F}=1-{\overline{\delta k_{i}}}^{F} / \overline{\delta k_{i}}$. The two fits agree across the scaled parameter space for $\eta_{\gamma}=0$ to within $4 \%$. Figure 7 also shows the relative difference, in this case as a function of energy spread and space charge

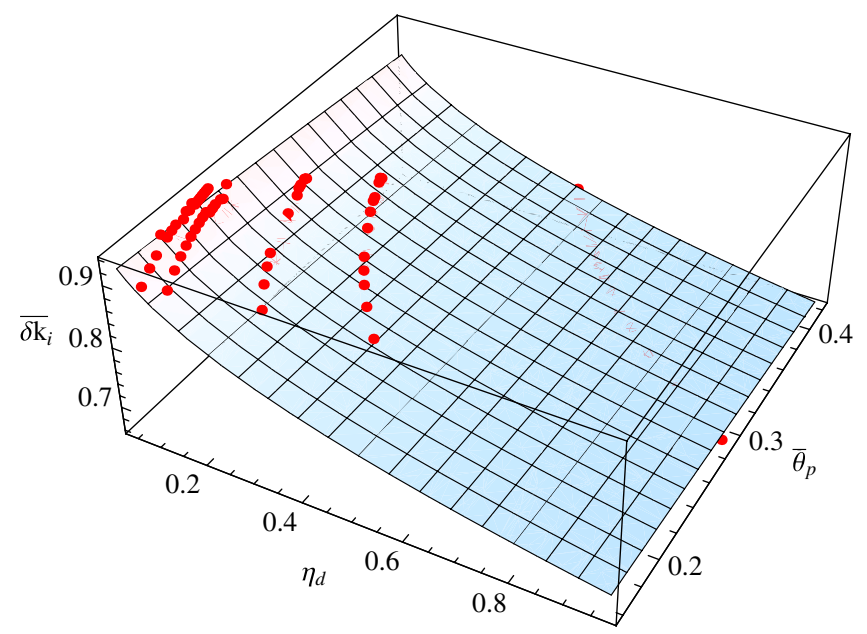

FIG. 5. Analytic fit contour and simulated data points for $\eta_{\gamma}=0$.

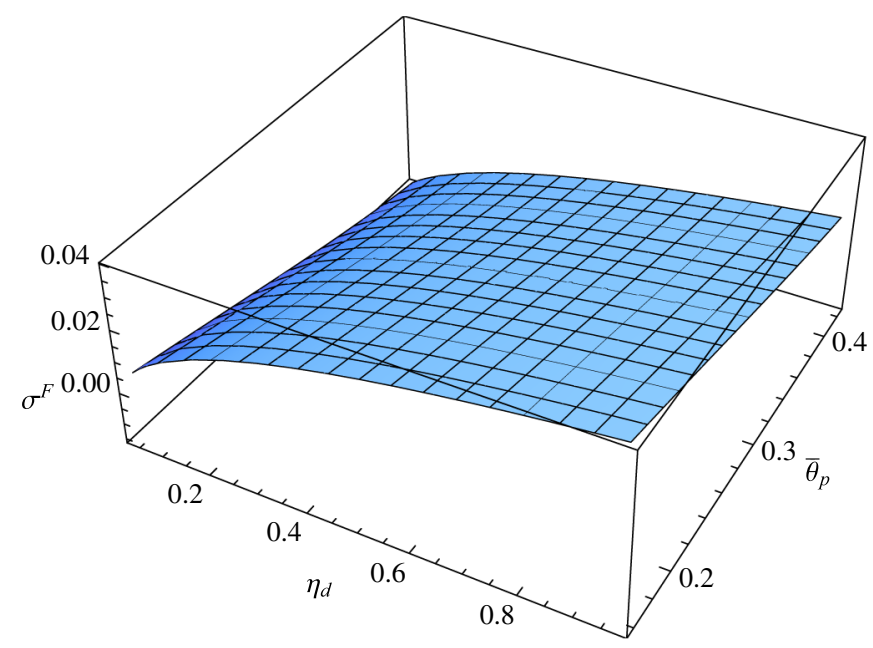

FIG. 6. Relative difference between the analytic and simulated fits for $\eta_{\gamma}=0$.

for $\eta_{d}=0.5$. This plot indicates that the two fits are in better agreement as the energy spread increases. Therefore, the $4 \%$ relative difference seen in Fig. 6 where $\eta_{\gamma}=0$ is the largest error found across the scaled parameter space.

It is worth noting that, as the diffraction parameter increases, the difference between the analytic and simulated fits increases as well. This can be seen in Fig. 5 where the analytic contour lies slightly above the simulated data values for large values of the diffraction parameter. This illustrates the limitations of the theory as the FEL system becomes more three dimensional in nature. It occurs because, as the diffraction parameter increases and the FEL becomes more sensitive to $3 \mathrm{D}$ effects, the transverse mode profile of the FEL light also increases. When the optical mode becomes larger than the e-beam, the shape of the profile begins to differ from that of the simple Gaussian assumed in the single-mode analytic model. This behavior

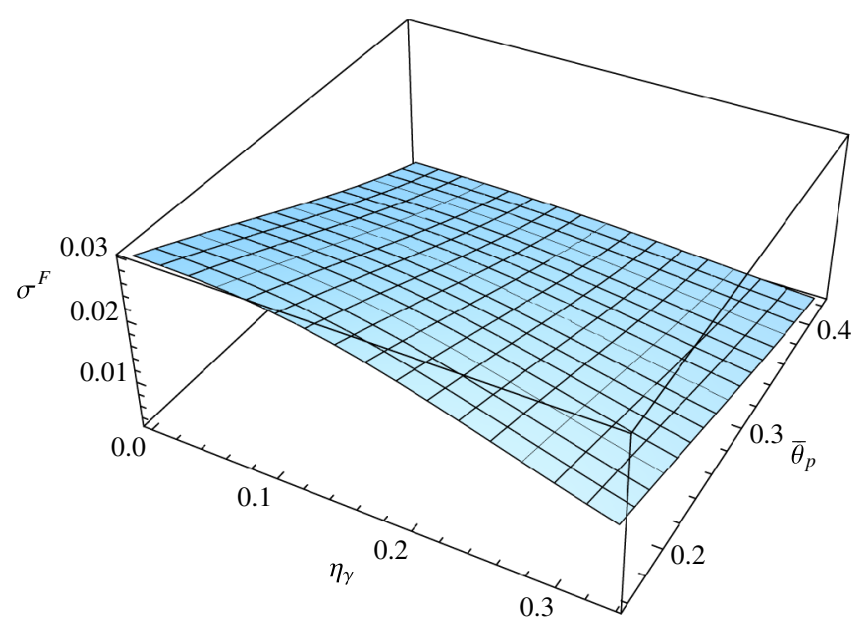

FIG. 7. Relative difference between the analytic and simulated fits for $\eta_{d}=0.5$. 


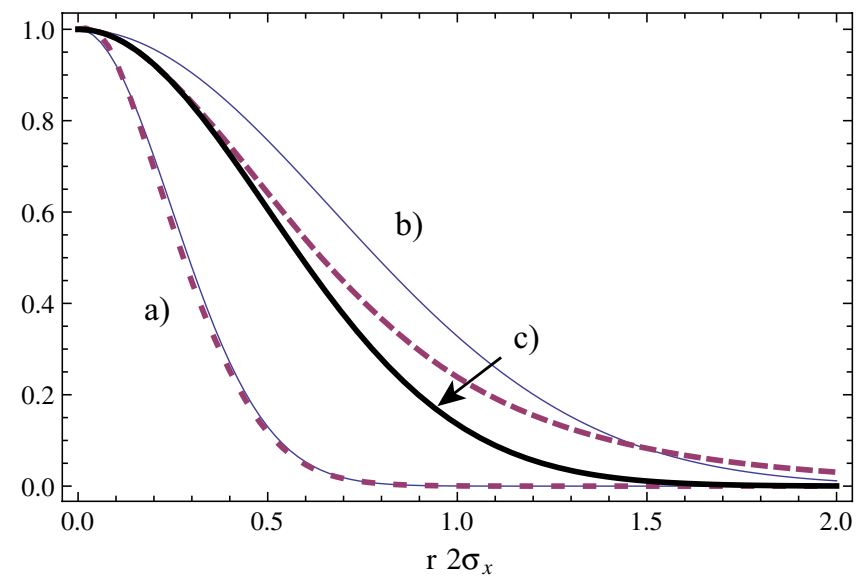

FIG. 8. Comparison of the transverse normalized intensity mode profile between simulation and theory for two cases: (a) a quasi-1D scenario with $\eta_{d}=0.04, \eta_{\gamma}=0, \bar{\theta}_{p}=0.23$ where the mode profile is contained within the e-beam, and (b) a 3D scenario with $\eta_{d}=0.97, \eta_{\gamma}=0, \bar{\theta}_{p}=0.28$ where the edges of the mode profile lie outside the e-beam. GENESIS results are represented as dashed lines while the solid, blue lines represent results from theory for both cases. The e-beam is shown in black (c).

is depicted in Fig. 8 where the theoretical and simulated optical profiles for two different regimes are compared. For small $\eta_{d}$ the system tends to be more 1D and the optical mode is well inside the e-beam. The agreement is excellent in this case, both in the predicted optical spot size and in the gain length. But as $\eta_{d}$ approaches unity, the large optical mode calculated from GENESIS clearly has richer structure, and does not precisely match the profile predicted from theory. This occurs because the region of the laser field near the e-beam center becomes narrowed due to gain compared to the region farther from the axis. The result is a sharper profile. The inability of the single Gaussian mode description to precisely match the optical profile for strongly 3D FELs is the primary source of the few percent error between the single-mode solutions and results from simulations for the predicted gain length. We note that future work could strive to eliminate this small error by instead using a non-Gaussian mode better suited to describe the pinched optical profiles of strongly 3D FELs.

It is also interesting to note that the agreement between simulated and analytic fits actually improves as both the space charge and the energy spread increase. This can clearly be seen in Fig. 7, where the relative difference between the analytical and simulated fits is shown as a function of $\eta_{\gamma}$ and $\bar{\theta}_{p}$ for $\eta_{d}=0.5$. Figure 9 shows that the agreement extends to the predicted profile where, despite a near doubling of the space-charge contribution, the profiles closely match. This illustrates that the presence of strong space-charge effects are well modeled by the theory presented.

We note that the numerical fit to the GENESIS data points, $\overline{\delta k}_{i}^{F}$, was omitted. We chose not to include this result for a

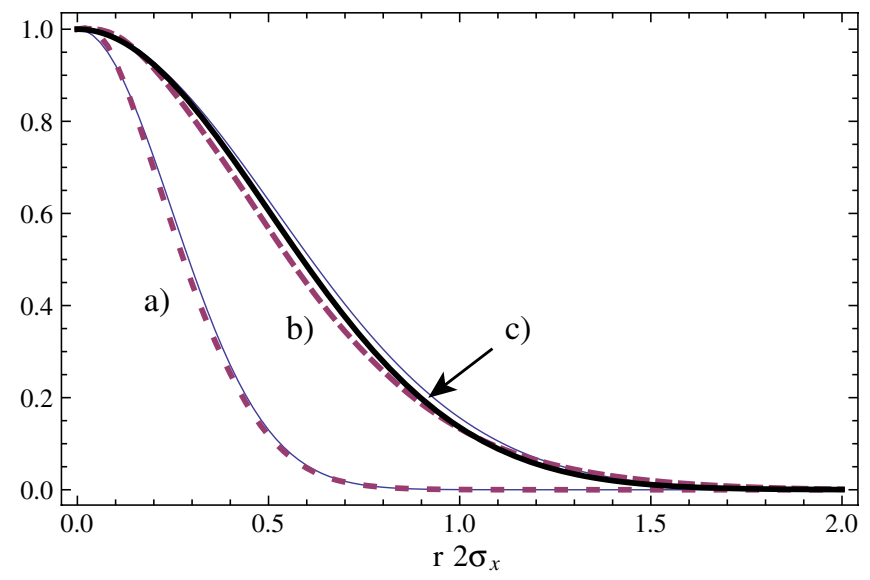

FIG. 9. Comparison of the transverse normalized intensity mode profile between simulation and theory for two cases: (a) $\eta_{d}=0.04, \eta_{\gamma}=0, \bar{\theta}_{p}=0.23$ where the mode profile is contained within the e-beam, and (b) $\eta_{d}=0.45, \eta_{\gamma}=0$, $\bar{\theta}_{p}=0.42$ where the mode profile approximately matches the e-beam. GENESIS results are represented as dashed lines while the solid, blue lines represent results from theory for both cases. The e-beam is shown in black (c).

variety of reasons. The power fit that was performed does not yield a unique solution, and the coefficients that are recovered from the fit are subject to multiple factors. These factors include the minimization method, the function used to minimize the residuals, and the constraints applied to initial parameter values. Regardless of these variables, every fit that was performed yielded similar results, where the agreement with the analytic fit was accurate to within at most $\sim 4 \%$. Also, the probed parameter space from the GENESIS simulations did not span the entire space where the analytic model is applicable, as evidenced in Table I. In addition, GENESIS includes only longitudinal space charge and does so on a Fourier decomposition basis. The inclusion of more Fourier coefficients in the simulation increases the accuracy of the space-charge calculation at the expense of computing time and resources. We found that the power fits obtained through simulation were in better agreement with the analytic model as more Fourier coefficients were included. For these reasons, we chose to quote only the results of the analytic fit.

\section{CONCLUSIONS}

We have presented a useful fit function similar to that of Xie that can be used to quickly calculate the gain length of the dominant three-dimensional optical mode of a FEL in the presence of uncorrelated energy spread, diffraction, and space charge. The formula was obtained from numerical solutions to the general 3D integro-differential FEL equations in the single, fundamental mode limit using a variational approach. Results have been compared to detailed numerical simulations using the 3D code GENESIS and show excellent agreement overall, with a maximum 
relative error of $4 \%$ attributed to a systematic issue inherent in the single Gaussian mode approximation for strongly $3 \mathrm{D}$ systems.

\section{ACKNOWLEDGMENTS}

The authors would like to thank G. Andonian for many helpful discussions. This research is supported by grants from Department of Energy Contracts No. DEFG02-07ER46272 and No. DE-FG03-92ER40693 and Office of Naval Research Contract No. N00014-06-1-0925.

[1] R. Bonifacio, C. Pellegrini, and L. M. Narducci, Opt. Commun. 50, 373 (1984).

[2] M. Xie, Nucl. Instrum. Methods Phys. Res., Sect. A 445, 59 (2000).

[3] P. Emma et al., Nat. Photon. 4, 641 (2010).

[4] E. Jerby and A. Gover, IEEE J. Quantum Electron. 21, 1041 (1985).

[5] E. Jerby and A. Gover, Phys. Rev. Lett. 63, 864 (1989).

[6] H. Freund, Nucl. Instrum. Methods Phys. Res., Sect. A 331, 496 (1993).

[7] A. Murokh et al., Phys. Rev. E 67, 066501 (2003).

[8] J. F. Schultz, M. J. Lavan, E. W. Pogue, and T. W. Meyer, Nucl. Instrum. Methods Phys. Res., Sect. A 318, 9 (1992).

[9] S. Benson et al., in Proceedings of the 26th International Free Electron Laser Conference, Trieste, Italy (2004), pp. 229-232.
[10] T. Watanabe et al., in Proceedings of the 27th International Free Electron Laser Conference, Palo Alto, CA (2005), pp. 320-323.

[11] D. Nguyen, in Proceedings of Linear Accelerator Conference 2006, Knoxville, Tennessee (2006), pp. 205-207.

[12] G. Geloni, E. Saldin, E. Schneidmiller, and M. Yurkov, Nucl. Instrum. Methods Phys. Res., Sect. A 554, 20 (2005).

[13] A. Marinelli and J. B. Rosenzweig, Phys. Rev. ST Accel. Beams 13, 110703 (2010).

[14] L. Serafini and J. B. Rosenzweig, Phys. Rev. E 55, 7565 (1997).

[15] E. L. Saldin, E. A. Schneidmiller, and M. V. Yurkov, Nucl. Instrum. Methods Phys. Res., Sect. A 475, 86 (2001).

[16] E. L. Saldin, E. A. Schneidmiller, and M. V. Yurkov, Opt. Commun. 97, 272 (1993).

[17] E. Hemsing, A. Marinelli, S. Reiche, and J. Rosenzweig, Phys. Rev. ST Accel. Beams 11, 070704 (2008).

[18] S. Reiche, Nucl. Instrum. Methods Phys. Res., Sect. A 429, 243 (1999).

[19] M. Xie and D. A. G. Deacon, Nucl. Instrum. Methods Phys. Res., Sect. A 250, 426 (1986).

[20] J. Murphy, C. Pellegrini, and R. Bonifacio, Opt. Commun. 53, 197 (1985).

[21] A. Gover and P. Sprangle, IEEE J. Quantum Electron. 17, 1196 (1981).

[22] G. T. Moore, Nucl. Instrum. Methods Phys. Res., Sect. A 239, 19 (1985). 